\title{
Du mot « catégorique » dans Les Géorgiques (1981) de Claude Simon
}

\section{Marie-Christine Mourier}

\section{(2) OpenEdition}

1 Journals

Édition électronique

URL : http://journals.openedition.org/ccs/902

DOI : $10.4000 /$ ccs.902

ISSN : 2558-782X

Éditeur :

Presses universitaires de Rennes, Association des lecteurs de Claude Simon

\section{Édition imprimée}

Date de publication : 31 mai 2014

Pagination : 85-96

ISBN : 9782753533387

ISSN : $1774-9425$

\section{Référence électronique}

Marie-Christine Mourier, " Du mot « catégorique » dans Les Géorgiques (1981) de Claude Simon », Cahiers Claude Simon [En ligne], 9 | 2014, mis en ligne le 22 septembre 2017, consulté le 25 avril 2019. URL : http://journals.openedition.org/ccs/902; DOI : 10.4000/ccs.902 


\title{
DU MOT "CATÉGORIQUE " DANS LES GÉORGIQUES (1981) DE CLAUDE SIMON
}

\author{
Marie-Christine MOURIER \\ Université Lille 2, laboratoire Calhiste (université de Valenciennes)
}

Aragon attaque la longue préface aux Yeux d'Elsa, intitulée Arma virumque cano, par ce passage sur ce que j'appellerai le charme de la faute:

J'avais à l'âge où l'on apprend à aimer les poèmes, été singulièrement frappé par ces vers de Rimbaud

Mais des chansons spirituelles

Voltigent partout les groseilles

tels qu'ils figuraient sous le titre Patience (D’un été...) dans l'édition Vannier. On veut aujourd'hui (édition critique, Mercure de France) qu'ils se lisent

Voltigent parmi les groseilles

et sans doute qu'il en est ainsi. Mais je ne puis refaire le chemin parcouru, et pour moi, tant que je vivrai, je lirai voltigent partout... avec cet étrange transitif du verbe voltiger qu'on peut me dire être une faute, et que je persiste à considérer comme une beauté ${ }^{1}$

Et nous savons que, si l'on excepte l'épigraphe, la première phrase du Fou d'Elsa est: "Tout a commencé par une faute de français ${ }^{2}$."

La faute jouerait un rôle de déstabilisation de la lecture qui permettrait la rêverie et ouvrirait à de nouvelles strates du texte: elle aurait une fonction poétique. Le mot jouerait dans le texte comme le bois peut jouer dans un meuble et le faire travailler. C'est un peu ces sentiments de déstabilisation et d'ouverture à un sens autre que j'ai éprouvés en lisant dans Les Géorgiques ${ }^{3}$ cette étrange description:

1. Les Yeux d'Elsa, "Arma virumque cano", Seghers, Paris, 1979, p. 9. Première parution mars 1942 aux Cahiers du Rhône.

2. Le Fou d'Elsa, Gallimard, 1963, p. 11. Aragon commet d'ailleurs lui-même une étrange faute dans

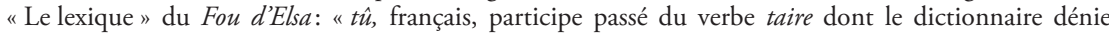
l'existence au masculin ", p. 450.

3. Les Géorgiques, (abréviation G), Minuit, Paris, 1981. Les références de pages renverront à cette édition. 
un de ces endroits où les trains ne s'arrêtent d'habitude jamais, en pleine campagne, et qui leur apparaît soudain comme un fragment figé, catégorique, de ces espaces sans véritable particularité, uniformisés par la neige... ( $G$, p. 84)

Le mot « catégorique » tranquillement glissé par Simon résiste au sens et suspend la lecture; il donne à rêver. Il n'y a pas de faute à proprement parler mais quelque chose ne va pas; l'usage de cet adjectif pour caractériser un paysage est anormal. C'est une forme d'hapax sémantique, un usage inventé par et réservé au seul Claude Simon. "Catégorique ", si l'on s'en rapporte au Grand Robert, équivaut à "relatif aux catégories", au sens philosophique et aristotélicien du terme et s'entend, par extension, comme "qui n'admet aucune objection". Mon sentiment d'étrangeté a été renforcé lorsque j'ai rencontré à nouveau ce mot dans le roman, dans un contexte apparemment tout autre, celui d'un souvenir du narrateur, lié aux séances de cinéma de l'enfance. Le qualificatif refait en effet surface, dans le chapitre III des Géorgiques, lorsqu'il est question des gitans censés occuper traditionnellement les premiers rangs de cette salle de cinéma.

[L]a conservation irrécusable et catégorique sous forme humaine de la violence à l'état pur: puis il y fut le garçon - c'est-à-dire plus un garçon alors, devenu un homme par une brusque mutation en l'espace d'une fraction de seconde, projeté aussi démuni qu'un nouveau-né dans ce qui est pour ainsi dire la face cachée des choses... ( $G$, p. 214-215)

Sans doute est-ce la violence dont les gitans sont porteurs qui est ici fixée par l'adjectif mais le texte glisse dans une seule et même phrase des gitans à la guerre, c'est-à-dire à ce que Simon nomme la "face cachée des choses". Le passage se fait par les deux points d'une ouverture ou d'une explication, d'un dépliement. La "violence à l'état pur" que le narrateur découvrira à l'œuvre en mai 40 relie les deux plis.

Tout ceci a donc à voir avec la guerre. Dans le premier cas, de jeunes soldats débarquent d'un train, dans le second cas, la phrase même accouche d'un basculement dans la guerre. Ajoutons que l'on découvre ce mot dans deux autres romans, L'Acacia (1989) et Le Jardin des Plantes (1997); son surgissement s'y fait à des nœuds particuliers des textes, et surtout systématiquement avec un sens plus que problématique. C'est encore dans le contexte d'un brutal changement d'environnement opéré par la guerre, celui que favorise le voyage nocturne en chemin de fer des jeunes mobilisés, que le terme "catégorique " sera utilisé dans le chapitre VI (27 août 1939) de L'Acacia:

comme si en moins de vingt-quatre heures il était brusquement passé non seulement du soleil au froid, mais d'un univers normal (y compris les foules et les femmes en pleurs) à un monde endeuillé, sévère, catégorique ${ }^{4} \ldots$

4. L'Acacia, Minuit, Paris, 1989, p. 200. 
Les adjectifs "endeuillé ", et "sévère " mènent donc à " catégorique ». Si " endeuillé " surprend, c'est qu'il ne peut être que proleptique, quant à " sévère ", il garde, par son étymologie latine (severus), le lien avec les puissances de la nuit.

Dans Le Jardin des Plantes, réécrivant la rencontre du 16 mai 1940 entre le Général Gamelin, les dirigeants français et Winston Churchill, le romancier, au moment où il évoque "le Général en chef » cessant de parler et donc de dire la défaite, décrit le chevalet sur lequel est posée une carte d'état-major. Ce meuble, Simon le qualifie également d' "irrécusable ", de " catégorique »:

ce chevalet qui au milieu de la débauche de stucs, $[\ldots]$ avait l'air d'un corps étranger $[\ldots]$ comme si dans ce décor $[\ldots]$ la guerre elle-même avait grossièrement fait intrusion, projeté là cet indécent, sommaire et catégorique meuble d'école, irrécusable $5 .$.

Il semble qu'il faille s'approcher à tâtons, par la série adjectivale, d'une vérité. C'est le terme "catégorique " qui conclut l'énumération des adjectifs antéposés. Aucune place ici à une variation des possibles; "Indécent » introduit une notation morale qui s'explique sans doute par le contraste entre la banalité du meuble scolaire et l'inhumanité du contexte guerrier. "Sommaire " peut renvoyer à la simplicité du support sur lequel est étalée la carte d'état-major, mais suggère aussi le passage brutal dans le monde de la défaite. Tout ce lexique dit la radicale étrangeté du réel.

Mais revenons maintenant aux Géorgiques et à la première occurrence du qualificatif rencontrée, en tentant de situer l'avant du texte.

\section{L'AVANT}

Le passage dans lequel le mot intervient, pour la première fois, fait partie du deuxième chapitre du roman. Simon a mis précédemment en place un filet serré mêlant les histoires du conventionnel, du cavalier, de l'enfant, du visiteur de la demeure ancestrale et d'Orwell. Tout est déjà dit. Tous les événements de 40, sur lesquels l'œuvre n'a cessé et ne cessera de faire retour, sont posés soit directement comme les traversées de la Meuse, la fuite avec ses différents arrêts, l'embuscade de Cousolre et l'attaque subie à Lez Fontaine, la file des prisonniers... soit indirectement, puisque pour les événements succédant à l'embuscade, Simon, en quelque sorte, renvoie à la Route des Flandres (1960). Rien n'est dans l'ordre mais tout est chronologiquement précisément installé et, pour la semaine de mai 40, interviennent des notations concernant l'heure de certains événements. Tout est présenté comme si le moment d'expulsion du train relevait d'un effet de convergence des tracés préalables: on songe au dessin de l'œil "démesurément agrandi » de Ledoux, peint en fond de scène 
du théâtre de Besançon, deux fois mentionné dans le texte ${ }^{6}$ ( $G$, p. 27 et p. 75) concentrant tout en un point focal. La structuration du récit fait que l'épreuve du cataclysme installée dans le premier chapitre accompagne tout ce qui sera évoqué et décrit ensuite. Le récit sera placé sous le signe de cette tonalitélà. Or, ce qui a été dit est que ce qui a été vécu pendant cette semaine de mai 40 - l'expérience - est de l'ordre de l'incompréhensible:

Aussitôt après avoir écrit cette phrase il se rend compte qu'elle est à peu près incompréhensible pour qui ne s'est pas trouvé dans une situation semblable et il relève sa main. (G., p. 47)

Lors des attaques de Lez Fontaine et de Cousolre, Simon est en effet passé de l'autre côté du mur du symbolique, il a brutalement découvert « la face cachée des choses ", le monde in-humain. L'utilisation des concepts lacaniens de réel, symbolique et imaginaire ${ }^{7}$ est, on le voit ici, particulièrement opérante ${ }^{8}$ pour comprendre ce qui est en jeu; il ne s'agit pas de soumettre le texte de Claude Simon à une grille psychanalytique étroite mais de saisir cette articulation entre découverte du réel - lors de cette semaine de mai 40 -, et création. Claude Simon a vécu un cataclysme. Brutalement, rapidement, la réalité s'est décomposée, est partie en morceaux.

Le bref combat de l'embuscade dans laquelle est tombé l'escadron semble terminé. Tout est maintenant silencieux. Au bout d'un moment il voit une mosaïque de polygones irréguliers de différentes dimensions, gris clair, gris bleuâtre, crayeux, ocre ou roses. Il est à quatre pattes sur le sol. ( $G$, p. 51$)$

Une séparation existait entre le monde et l'immonde et il semble que l'événement, l'embuscade qui a anéanti l'escadron, ait fait voler en éclats cette cloison, il a secoué la réalité comme un "vulgaire décor de toile" ( $G, \mathrm{p} .130)$. À l'évidence, le bruit joue un grand rôle dans ce bouleversement total vécu par le corps et l'esprit. Le silence qui lui succède indique que le monde, celui du

6. Cette figure avait été analysée comme ouverture sur «l'autre scène» dans Lucien Dällenbach, Claude Simon, "Les contemporains », Le Seuil, 1988, p. 51.

7. On se souvient que la théorie lacanienne des trois ordres (Réel, Symbolique, Imaginaire) s'intéresse au statut de l'être parlant (le parlêtre). L'Imaginaire à partir de la constitution du corps et de l'image de soi, image trompeuse, est le registre du moi avec ses leurres, méconnaissances et aliénations. Le Symbolique est le registre de la loi humaine en tant qu'englobant toute l'activité humaine. Il se met en place chez le sujet à partir du manque. Il relève du langage comme structure. Il est fait d'éléments différenciés. Il préexiste au sujet et le constitue. Le Réel c'est ce que l'intervention du symbolique a chassé de la réalité. Il est l'inhumain, l'innommable, l'indifférencié. Les trois ordres sont liés. Je choisis d'écrire Réel sans majuscule dans le texte.

8. Lire à ce sujet Claude Simon Writing the visible de Célia Britton, Cambridge University Press, 1987. On regrettera que cet ouvrage n'ait jamais été traduit. Lire aussi Marie-Christine Mourier: "Claude Simon, quand le surgissement du réel oblige à une poétique ", Revue Électronique de Littérature Française, 4 (1), 2010, p. $27-42$. 
narrateur, a été cassé ${ }^{9}$. La matière brutale se révèle ${ }^{10}$. En lieu et place de cette réalité mise en morceaux, un plein s'impose. Alors qu'il progresse à nouveau à cheval, dans un printemps indifférent à l'événement, le narrateur a l'illusion d'être séparé de ce qui l'entoure par une épaisse couche de verre; le monde, devenu autre, ne se laisse plus rejoindre par le biais des sensations habituelles:

Les sons des cloches attachées à leurs cous et les aboiements du chien parviennent affaiblis et avec un moment de retard, comme s'ils avaient à traverser une épaisse couche de verre, une pellicule à la fois transparente et opaque qui isolerait le visage du monde extérieur. ( $G$, p. 51)

Manifestement, Simon retranscrit au plus près ce qu'il a ressenti en cette étape décisive de son existence. Il tentera, en redonnant une explication plausible des faits, de faire repasser l'impression dans l'ordre de l'humain mais les explications qui seront dispersées dans le contexte ultérieur et dans les autres romans seront tout à la fois récurrentes et incohérentes. Il sera question de matériaux qui cloisonnent, "cellophane " ou "verre "... C'est sans doute L'Acacia qui tentera de restituer au plus près la sensation éprouvée. Le point commun de toutes ces séquences, c'est bien la perception par le narrateur d'un étrange plein, d'un monde figé remplaçant une réalité partie en morceaux. Le cataclysme paraît avoir fait passer le cavalier dans un univers sans fissures. Simon aura de nouveau recours à l'idée donnée par le mot " figé ${ }^{11}$ ", ici décliné en verbe conjugué pour tenter de rendre compte de l'entrée dans cet espace constitué d'opacité; le qualificatif fait partie, on l'a vu, de l'environnement lexical de l'adjectif « catégorique ». Le monde est devenu chaos puis le visible s'est opacifié. Rappelons que la description du cantonnement qui suit le chapitre 2 sera marquée, elle aussi, par la modalité du plein. C'est le froid, l'on s'en souvient, qui devient alors matière, " une matière transparente, coupante comme du verre, à la fois compacte et fluide» $(G$, p. 102).

Quelques lignes avant la description du paysage qui nous intéresse, Simon se situe d'ailleurs explicitement dans la ligne du cataclysme:

9. Simon traquera dans le reste du roman la force de ce qui a été éprouvé en tentant à nouveau de le transcrire; le texte qui suit est postérieur à la première occurrence du mot catégorique: "quelque chose d'obscur, marron, se fracassant, une dégringolade de triangles, comme les innombrables fragments d'une vitre volant en éclats (quoique l'affaire se passe en pleine campagne, sans aucune maison aux alentours), les bordures des triangles lumineuses, éblouissantes, le bruit [...] semblable à un tintamarre de verre brisé... " ( $G$, p. 177-178). Italiques de Claude Simon.

10. Simon, dans sa réécriture d'Hommage à la Catalogne, utilisera presque les mêmes mots: « (et plus qu'un fracas: l'air, la terre secoués, déchiquetés) qui, même si on en a déjà l'expérience, frappe celui qu’il assourdit de quelque chose de plus fort que la peur; l'horreur, l'ahurissement, le scandale, la soudaine révélation qu'il ne s'agit plus là de quelque chose à quoi l'homme ait tant soit peu part mais seulement la matière délibérée, sauvage, furieuse, indécente... ( $G$, p. 289). Jamais Orwell ne parle de matière, juste fugacement d'" horreur ".

11. «[T] out ce qui allait, à un signal donné, éclater, exploser avec la violence d'un cataclysme, mais attendant encore, s'effaçant provisoirement devant ce cataclysme d'une autre nature qui figeait aussi les coteaux...» $(G$, p. $133-134)$. 
Les brefs combats n'auront pas le temps d'apporter de modifications à cet état de choses.

Du fait de leur violence d'abord: la rapidité cataclysmique avec laquelle tout se déroulera, l'agression inouïe du bruit, l'absence d'ordres, la carence du commandement... (G, p. 81)

C'est aussi, avant la sortie même du wagon que sera intensifié ce rapport à la catastrophe par une désignation du réel très claire puisque le romancier choisit une définition quasi philosophique de la guerre:

Il éprouve intensément (le wagon à bestiaux, la destination inconnue, les chocs brutaux des roues aux cassures des rails, le froid enfermé, statique, serré comme un écrou, la solitude à plusieurs) un sentiment d'agression (pas les hommes, la guerre: les choses, l'étant). ( $G$, p. 80)

Le réel de la matière est alors redonné par le rappel d'un souvenir enfantin clairement associé à l'impression précédente:

Déjà enfant dans les toilettes ${ }^{12}$ d'un train express: il appuie sur la pédale et par la lunette ouverte de la cuvette des W.C. il voit filer dans un tapage brutal de choses concassées qui lui saute au visage les traverses et les cailloux du ballast. Froid puant qui semble soufflé d'en bas. Coulées de merde et papiers de soie détrempés, ridés, plaqués au cylindre gluant du conduit. ( $G$, p. 80)

Encore une fois, le bruit, avec ici la présence des choses concassées, contribue au traumatisme.

L'apparente déstructuration du roman est donc une mise en perspective. Tout est revu et réécrit dans cette aura de l'après. Tout est lu aussi dans cette perspective. Tout le roman sera orienté par la nécessité de dire l'indicible et de refuser les catégories portées par les clichés anciens associées à l'idée de conflit guerrier. Ce qui suivra l'irruption catégorique de l'événement sera donc présenté non comme une découverte mais comme une construction susceptible de donner un sens englobant au parcours des soldats dans ce bout du monde. Voyons donc maintenant l'après.

\section{L'APRÈS}

Le passage qui nous intéresse ouvre, en effet, à l'évocation d'un long cheminement/errance que l'on retrouve dans deux autres romans, La Route des Flandres et L'Acacia. Seulement, contrairement aux autres épisodes de la guerre précisément situés et localisés, ces cheminements/errances bougent nettement d'un texte à l'autre, signe qu'ils ne sont accrochés à aucun événement précis mais qu'ils ont une autre fonction, celle peut-être de signifier la guerre de 40, en particulier dans ses manquements au niveau du haut commandement et dans la désintégration qui s'en est suivie. Dans Les Géorgiques ce cheminement/errance nous dirige vers un lieu déceptif puisque ce qui sera décrit est

12. On se souvient que dans L'Herbe (voir en particulier la page 93 de l'édition originale), ce sont les bijoux de Sabine qui passent par le tuyau d'évacuation des toilettes du train. 
le cantonnement précédent; il signe la perte d'identité et la désintégration de toute unité, de toute l'Unité, il dit l'isolement total des cavaliers. Tout ce que Simon donne comme éléments sur la solitude des cavaliers, «la solitude à plusieurs " est une recherche d'explication humaine de la violence à venir mais déjà là dans le texte. Chez Barbusse ${ }^{13}$ par exemple, qui raconte bien pire, il n'est jamais relaté un traumatisme du type de celui vécu par le narrateur car le groupe de soldats est soudé: malgré l'horreur vécue, leur univers reste humain. Simon montre comment le traumatisme a été préparé par ce déficit de communauté et c'est là un des enjeux de la séquence de cheminement/errance. La violence du cataclysme a été ressentie par un homme seul, parce qu'il était seul. Entre un avant qui est déjà un après et un après qui est déjà un avant, se place donc cette description rapide d'un paysage catégorique.

Le texte se déroule au présent: temps de l'instant, temps du présent de la rédaction et de la lecture, temps qui tente de lire l'événement et peut-être de le donner à revivre. L'arrivée se fait néanmoins encore au pluriel. Les cavaliers forment un groupe, au moins par le biais du pronom "ils». Mais cette arrivée est présentée en deux modulations, chacune placée avant la tentative de description du lieu; les cavaliers sont d'abord décrits comme sautant du wagon sur le ballast, donc agissant physiquement. Puis vient une tentative d'explication sur "l'affaire ". Le saut sur le ballast débouche, au bout de ces pérégrinations du texte, sur la caractérisation du lieu. Lieu qui est à la fois banal et distinct des lieux humains habituels, des lieux familiers. Le texte joue d'un effet de dramatisation pour dévoiler le paysage qui apparait soudain aux soldats; avant d'avancer une caractérisation, Simon précise que la banalité de l'endroit joue à contresens de ce que les soldats vont vivre:

il semble que jouèrent pour ainsi dire à contresens l'extrême banalité et la platitude des circonstances, à l'image de la banalité de l'endroit où s'est immobilisé le train... (p. 84)

À contresens, car l'événement lui sera tout sauf banal. Le lieu est donc banal et catégorique, banal et figé, banal et naturel. C'est l'événement qui à rebours va révéler le lieu:

un de ces endroits où les trains ne s'arrêtent d'habitude jamais, en pleine campagne, et qui leur apparaît soudain comme un fragment figé, catégorique, de ces espaces sans véritable particularité, uniformisés par la neige, entrevus toute la journée par la fente verticale de la porte mal jointe. $(G$, p. 84$)$

Le monde est là, banal, sans trait saillant, à l'image de ce qu'ils ont vu et, en même temps, il est d'une autre sorte. Le paysage n'a pas de particularité, car dans la différence se loge le symbolique. Catégorique est ce paysage car il est sans fissure. Il est irréductible, radical dans son altérité, imposant son évi-

13. Henri Barbusse, Le Feu, Journal d’une escouade, Payot, coll. « Petite Bibliothèque Payot », 2012. 
dence, résistant à la nomination, figé dans la matière comme le seront les paysages du cataclysme. Le mot " catégorique " scintille dans ce sens nouveau qui transcrit la tentative de dire l'ailleurs absolu. Dans la deuxième modulation de leur arrivée, les soldats sont d'ailleurs décrits d'une manière toute autre: ils ne sautent plus du wagon, ils sont expulsés comme sur une " planète »:

et pour ainsi dire expulsés, arrachés de leurs abris (les wagons à bestiaux parcourus de courants d'air mais constituant tout de même un espace clos), brutalement jetés, déposés comme à la surface d'une planète morte, dépeuplée et glacée. ( $G$, p. 84-85)

On peut en déduire que les deux modulations de la sortie du wagon sont deux tentatives d'approche de l'indescriptible; la première avait saisi des soldats en mouvement, dans l'action, mais la seconde, portée par la dynamique textuelle, a débouché sur la métaphore de l'expulsion, expulsion qui peut être naissance ou défécation. Expulsion qui signe la passivité, le "ça arrive ". Les soldats sont expulsés dans un monde inhumain, une planète morte. Il n'y a pas ici d'Orion géant pour cacher, par le biais de la figuration et de la nomination, l'intraitable du cosmique. "Les étoiles sont intégralement réelles, en principe, il n'y a rien chez elles absolument rien qui soit de l'ordre d'une altérité à ellesmêmes, elles sont purement et simplement ce qu'elles sont ${ }^{14}$." Dans le même temps tout le texte, on le voit, tente de redonner du sens à l'insensé, de passer du contresens au sens et surtout de nommer. Mais pour dire l'innommable, il lui aura fallu passer par l'invention lexicale sans pour autant avoir fait le tour de ce qui a été éprouvé car l'épreuve est indicible, et par là même, paradoxalement source langagière.

\section{LES GITANS}

Et, très curieusement, sur cette planète dépeuplée, Simon fait réapparaître un groupe humain, les gitans tout d'abord, puis les Espagnols. La seconde occurrence du mot "catégorique » intervient en effet au cour d'une description très étrange, celle des gitans aux premiers rangs de la salle de cinéma fréquentée dans l'enfance. Étonnons-nous d'abord de ce que Simon écrit:

(...confus remue-ménage d'une horde barbare innombrable) [...] pareilles (les tribus) à ce qu'elles étaient déjà à l'origine des temps, elles, leurs femelles squelettiques ou énormes, parfois même difformes, éléphantines, comme si les lois immémoriales de l'errance et du pillage les avaient façonnées une fois pour toutes aux seules images de la famine ou de la ripaille, aussi impassibles, aussi insensibles aurait-on dit, dans l'un ou l'autre des deux états, avec leurs profils desséchés d'oiseaux de proie... (G, p. 212-213)

Il est donc question de " tribus » et ce mot nous projette dans un temps autre que le nôtre - temps "incivilisé » contre temps civilisé -, un temps qui ne 
serait pas sensible au temps. Car le rapport que nouent ces êtres avec la matière via leurs corps n'est pas de l'ordre de la norme; il est d'ailleurs question de «femelles" et non de "femmes" comme si l'animalité était ici première. L'adjectif " éléphantines ", qui intensifie "énormes ", renvoie à une obsession de l'obèse propre à Simon: il désigne une forme de plein, une impossibilité $\mathrm{du}$ vide. Cette relation particulière au temps et à la matière fait de ce peuple de gitans un groupe aux pouvoirs occultes, lié au chaos, peuple qui a quelque chose à voir avec la confusion originelle :

investis de ces occultes pouvoirs qui leur conféraient le don non pas de jeter des sorts ou de prédire l'avenir mais en quelque sorte de le préfigurer, c'est-à-dire d'accélérer le temps, confondant passé présent et futur dans un même creuset, assimilant, transformant en objets archaïques, primitifs et démantibulés tout ce qu'ils approchaient ou touchaient, restituant au chaos, à la matière originelle (rouille, boue, pourriture - de même qu'ils pouvaient indifféremment réduire au même dénominateur commun de crasse, d'odeurs pestilentielles, de ruisseaux d'excréments, d'épaves ou de porcherie n'importe quelle habitation [...]) les produits les plus robustes ou les plus sophistiqués du monde qui les entourait... (G, p. 214)

Dans cette salle de cinéma, autour de cette tribu, Simon s'invente donc un contrepoint à l'autre descente aux enfers évoquée tout au long du roman, à savoir celle qui s'associe à l'Orphée et Eurydice de Gluck. C'est dans cette proximité avec la " horde » que se joue la rencontre fantasmée a posteriori avec la violence absolue, " catégorique » donc.

puis il y fut (le garçon - c'est-à-dire plus un garçon alors, devenu un homme par une brusque mutation en l'espace d'une fraction de seconde, projeté aussi démuni qu'un nouveau-né dans ce qui est comme pour ainsi dire la face cachée des choses...) ( $G$, p. 215)

La phrase qui place le mot opère à nouveau le basculement dans la guerre avec cette idée, souvent énoncée chez Simon, que les soldats sont aussi démunis que des nouveau-nés, nouveau-nés que Simon a placés quelques lignes plus haut dans les bras des gitanes et décrits comme " de petits morts" ( $G$, p. 213). Il n'est pas indifférent que ce passage d'un monde à l'autre se fasse par les deux points d'une ouverture. Très curieusement, Simon rêve un monde humain/ inhumain qui vivrait pourtant dans le réel, monde de la violence absolue, monde qui n'a jamais existé, monde d'une fausse préhistoire, monde des tribus et de l'excrément. Or cette rêverie d'une tribu humaine/inhumaine, nous la retrouvons à plusieurs reprises dans l'œuvre de Simon. Dans Les Géorgiques, la description de l'Espagne est de cet ordre:

sans se rendre compte qu'au fur et à mesure qu'il descend vers le sud il effectue dans le temps un parcours inverse, remonte à toute vitesse l'espace de plusieurs siècles pour être précipité dans un univers où aucune des notions, aucun des mots qui le constituent n'ont de sens... (G, p. 319) 
Il s'agit d'un monde "où des hommes sauvages combattent des bêtes sauvages... ", ( $G$, p. 320), un monde d'avant l'histoire humaine ou les humains adorent des idoles:

idoles voilées de noir, sanglantes, endiamantées, aux cœurs percés de poignards, comme les symboles conservés intacts non pas même de ce passé que d'autres avaient répudié en même temps qu'ils coupaient la tête de leurs rois, mais de quelque chose d'avant même les rois, comme si aux derniers confins d'un continent pendait une sorte de fruit desséché et ridé, oublié par l'histoire et rejeté, repoussé par la géographie, comme un récipient, une espèce de cloaque... (G, p. 320)

Il est encore question plus loin de "fanatique superstition des primitifs" ( $G$, p. 325). Orwell, l'écrivain qui a été étudiant à Oxford, a contrario est du monde des humains, des civilisés mais dans cette Espagne ainsi décrite, Simon imagine qu'il, Orwell, a découvert le monde de la matière:

(et plus qu'un fracas: l'air, la terre secoués, déchiquetés) qui, même si on en a déjà l'expérience, frappe celui qu'il assourdit de quelque chose de plus fort encore que la peur : l'horreur, l'ahurissement, le scandale, la soudaine révélation qu'il ne s'agit plus là de quelque chose à quoi l'homme ait tant soit peu part mais seulement la matière libérée, sauvage, furieuse, indécente... ( $G$, p. 289)

Rappelons que le narrateur, lui, se voit comme appartenant à un monde mi-humain/mi-animal lorsqu'il se décrit vivant, pendant l'hiver 39-40, au cantonnement:

projetés, comme hors de l'Histoire, ou livrés à quelque chose qui se situait au-delà de toute mesure [...] : l'état (temps, espace, froid) où devait être le monde à l'époque des cavernes, des mammouths, de bisons, et autres bêtes gigantesques chassées par des hommes gigantesques pour prendre leurs fourrures, boire leur sang chaud, au sein de gigantesques et inépuisables forêts. ( $G$, p. 119)

Dans ce monde in-humain révélé par le cataclysme, Simon s'invente donc des pré-humains, des tribus qui ont un rapport au temps et à la matière d'un autre ordre que le nôtre. Il les dotera même dans Le Jardin des Plantes de la capacité à dire le réel, à le nommer; il s'agira cette fois de nommer la montagne-Léviathan, celle qui signifie la Chine:

Aucun nom (ou peut-être dans des idiomes des dialectes incompréhensibles aux consonances étranges, sauvages, gutturales ou soyeuses comme peuvent l'être le cri d'un oiseau, d'un aigle, de quelque animal surnaturel pacifique et meurtrier- et, de fait, c'était à cela que ça ressemblait: non pas ce que l'on appelle habituellement des montagnes, une simple barrière faite d'inertes roches, d'aiguilles ou d'inertes névés, mais à quelque chose de vivant, assoupi et terrifiant, de la famille des sauriens ou des reptiles dont elle avait les replis, et qui devait tuer sans même se donner la peine de bouger ${ }^{15} \ldots$ )

Les guerriers, au contraire, relèveraient de l'humain; le guerrier symboliserait la victoire sur le réel, le fait de ne pas se laisser déstabiliser par l'événe- 
ment, de savoir l'affronter; il n'est évidemment pas question, dans ce cas, des incapables comme les généraux et autres officiers ayant manifesté leur incompétence, mais de ceux, comme le conventionnel L.S.M., qui sont à la hauteur de leur tâche ou du maréchal des logis Auguste Ostertag ${ }^{16}$. Les guerriers, déjà, dans Les Corps conducteurs (1971) étaient ceux qui affrontaient la forêt amazonienne, la forêt du réel. Simon, lui, de cette mauvaise rencontre, fait œuvre de création, invention d'un autre code. Le cataclysme lui aura fait découvrir un monde proprement indicible, à l'inverse de celui qui est figuré dans le dessin de l'incipit; on se souvient que l'artiste a "cherché, dans la scène proposée, à nettement différencier les différents éléments selon leur importance croissante dans son esprit... ", $(G$, p. 14). Le romancier avait d'ailleurs ordonné la description: " premièrement ", " deuxièmement "... et avait fait apparaitre, parmi les objets énumérés une carte, " une grande carte ancienne suspendue sur l'un des panneaux, avec sa rose des vents, ses chaînes de montagnes en formes de taupinières » et une mappemonde " entourée à l'équateur d'un anneau zodiacal et montée sur un socle à trois pieds ", $(G$, p. 14).

Le mot "catégorique" renvoie à un univers en rupture avec ces classements et ces socles à trois pieds. Sans jeu de mots, il n'entre pas dans ces catégories, il ne se range pas. "Le réel ne saurait s'inscrire que d'une impasse de la formalisation ${ }^{17}$ "; pour Lacan on ne peut parler du réel, plus exactement on peut tenter d'en parler, mais en le ratant toujours. Ce que Simon a à dire n'est pas de l'ordre des choses connues. Le dessin décrit figure, lui, des " anatomies stéréotypées " $(G$, p. 12), et il est question d'un code d'écriture: "il est évident que la lecture d'un tel dessin n'est possible qu'en fonction d'un code d'écriture admis d'avance par chacune des deux parties, le dessinateur et le spectateur " ( $G$, p. 13). Le mot "catégorique" n'est pas de cet ordre. Le code ne fonctionne plus. Déjà, dans La Bataille de Pharsale, la quête répétée de figurations anciennes de la guerre signifiait que le code était à réinventer. Dire l'indicible sans fissure, magma vitreux ou excrément menaçant, force à la fabrication d'un mot qui garde, dans le mystère de son sens, la résistance à être dit du réel. Dire l'indicible force à l'écart. Il s'oppose au monde stéréotypé présent jusque dans les rochers artificiels de la mise en scène d'Orphée et Eurydice. Il est porte d'entrée dans un monde inhumain, porte manquée dans le monde des tribus d'avant l'histoire humaine. Il est bouche d'ombre.

16. Il n'est que très rapidement question de ce dernier dans Les Géorgiques mais celui-ci, sans être nommé, est très présent dans La Bataille de Pharsale (1969); dans Le Jardin des Plantes, son patronyme sera donné, la citation à l'ordre de l'armée retranscrite (JP, p. 362).

17. Jacques Lacan, séminaire, livre XX, Encore, texte établi par Jacques-Alain Miller, Paris, Le Seuil, 1975, p. 85. 
La découverte cataclysmique force donc à l'invention d'un nouveau langage et ce, au-delà du "catégorique ». Car toute la langue de Simon est de cet ordre, elle énonce cet aller et retour entre le réel et les lopins, cette tension entre la sauvagerie et la terre que l'on veut marquer à toute force de son empreinte. Nous sommes loin du "rêve d'un règne étale du symbolique ${ }^{18}$ ». C'est l'invention d'une nouvelle langue, langue du tâtonnement, de l'énumération, de la parenthèse, langue de la fragmentation et du lien; ce retour au symbolique, au A de l'alphabet, se fait avec la jouissance de savoir qu'il sera toujours manqué car "qui croit nommer [...] le réel a, de fait, nommé tout autre chose $\mathrm{e}^{19}$ ". Nous sommes donc à la fois dans l'horreur et l'enchantement. Le chant naît de l'impossibilité à dire l'horreur et nous lecteurs en sommes fascinés car l'accrochage de notre propre langue a bougé.

18. Jean-Claude Milner, Les Noms indistincts, Paris, Le Seuil, 1983, p 34.

19. Ibid., p. 21. 